\title{
Numerical solution for a class of multi-order fractional differential equations with error correction and convergence analysis
}

\author{
Wei Han' ${ }^{1}$, Yi-Ming Chen ${ }^{1,2,3^{*}}$, Da-Yan Liư ${ }^{4}$ Xiao-Lin Li ${ }^{1}$ and Driss Boutat ${ }^{4}$
}

"Correspondence:

chenym@ysu.edu.cn

${ }^{1}$ School of Sciences, Yanshan

University, Qinhuangdao, P.R. China

${ }^{2}$ Loire Valley Institute for Advanced

Studies, Orléans, France

Full list of author information is

available at the end of the article

\section{Springer}

\begin{abstract}
In this article, we investigate numerical solution of a class of multi-order fractional differential equations with error correction and convergence analysis. According to fractional differential definition in Caputo's sense, fractional differential operator matrix is deduced. The problem is reduced to a set of algebraic equations, and we apply MATLAB to solve the equation. In order to improve the precision of numerical solution, the process of error correction for multi-order fractional differential equation is introduced. By constructing the multi-order fractional differential equation of the error function, the approximate error function is obtained so that the numerical solution is corrected. Then, we analyze the convergence of the shifted Chebyshev polynomials approximation function. Numerical experiments are given to demonstrate the applicability of the method and the validity of error correction.
\end{abstract}

Keywords: Shifted Chebyshev polynomials; Multi-order fractional differential equation; Error correction; Convergence analysis; Numerical solution

\section{Introduction}

Fractional calculus [1] is developing fast and its various applications are extensively used in many fields of science and engineering. It has been applied to chaotic systems $[2,3]$ and optimal control problems [4]. In [5], the authors derived the fractional Euler-Lagrange equation in terms of the Caputo fractional derivatives. Kumar et al. [6] analyzed FornbergWhitham equation pertaining to a fractional derivative with Mittag-Leffler type kernel. The authors of [7] investigated a time-fractional modified Kawahara equation through a fractional derivative with exponential kernel. In [8] Singh et al. presented a fractional epidemiological model and solved the solution of the problem by using an iterative method.

Fractional differential equation [9] is used to describe mathematical phenomena of many areas, such as rheology, damping method, signal processing, control theory, polymers, viscoelastic materials, and so on. Many researchers $[10,11]$ focus on the numerical treatments of fractional differential equation, such as homotopy analysis transform method [12, 13], iterative reproducing kernel Hilbert space method [14], artificial neural network approach [15], variational iteration method and its modification [16], Wavelet

(c) The Author(s) 2018. This article is distributed under the terms of the Creative Commons Attribution 4.0 International License (http://creativecommons.org/licenses/by/4.0/), which permits unrestricted use, distribution, and reproduction in any medium, provided you give appropriate credit to the original author(s) and the source, provide a link to the Creative Commons license, and indicate if changes were made. 
method [17-19], Bernstein polynomials [20], and fractional-order Legendre functions [21]. The authors of [22] researched space-time fractional Rosenou-Haynam equation. In [23] Baleanu et al. solved the time fractional third-order evolution (TOE) equation with Riemann-Liouville (RL) derivative.

Since multi-order fractional differential equations are applied in many fields, many scientists have begun to study the properties and numerical solutions of equations. Multiorder fractional differential equation [24] is one of the most important types of fractional differential equations. Authors of $[25,26]$ investigated the existence, uniqueness, convergence of the solution for multi-order fractional differential equation. Because there is no exact solution, most different numerical methods, such as stable fractional Chebyshev differentiation matrix [27], fractional-order operational method [28], spectral collocation methods [29], and so on, have been used to investigate the approximate solutions of multiorder fractional differential equation. The authors of [30] only researched the convergence effect of numerical solutions and exact solutions of equations. There is little literature with shifted Chebyshev polynomials to solve multi-order fractional differential equation and research error correction and convergence. In this paper, the numerical solutions of a class of multi-order fractional differential equations with error correction and convergence analysis are investigated. According to the function approximation theory and fractional differential operator matrix, the equation is transformed into algebraic equations. The correction solutions of multi-order fractional differential equation are investigated and the convergence of the shifted Chebyshev polynomials approximation function is analyzed. We do the correction for the numerical solution of low precision and obtain the absolute error of the correction solution, so that the accuracy of the numerical solution is improved.

In general, multi-order fractional differential equation is expressed as follows:

$$
D^{\alpha} u(x)=\sum_{i=0}^{k} y_{i} D^{\beta_{i}} u(x)+f(x), \quad x \in[0,1]
$$

with the initial conditions

$$
u^{(p)}(0)=d_{p}, \quad p=0,1, \ldots, n-1,
$$

where $n-1<\alpha \leq n$, the coefficient $y_{i}(i=0,1, \ldots, k)$ is constant, and $0<\beta_{0}<\cdots<\beta_{k}<\alpha$, $f(x)$ is a known function.

The rest of the paper is organized as follows: Sect. 2 introduces the definition of Caputo fractional derivatives and shifted Chebyshev polynomials. In Sect. 3, the function approximation theory is introduced. In Sect. 4, the process of error correction for multi-order fractional differential equation is introduced and the convergence of the shifted Chebyshev polynomials approximation function is analyzed. Section 5 deduces the fractional differential operator matrix based on shifted Chebyshev polynomials. Section 6 reduces the problem to a set of algebraic equations. In Sect. 7, the proposed method is applied to two examples. Conclusion is given in Sect. 8.

\section{Preliminary knowledge}

In this section, we give the definition of the Caputo fractional derivatives and shifted Chebyshev polynomials in [31]. 


\subsection{The Caputo fractional derivatives}

Definition 1 The Caputo fractional derivative operator $D_{x}^{\alpha}$ of order $\alpha$ is defined in the following form:

$$
{ }_{a}^{C} D_{x}^{\alpha} f(x)=\frac{1}{\Gamma(m-\alpha)} \int_{a}^{x}(x-T)^{m-\alpha-1} f^{(m)}(T) d T, \quad \alpha>0,
$$

where $m-1 \leq \alpha<m, x>0$.

For Caputo's derivatives, we have

$$
{ }_{a}^{C} D_{x}^{\alpha} C=0,
$$

where $C$ is a constant.

$$
{ }_{a}^{C} D_{x}^{\alpha} x^{m}=\frac{\Gamma(m+1)}{\Gamma(m+1-\alpha)} x^{m-\alpha}, \quad m \in N_{1}, m \geq\lceil\alpha\rceil,
$$

where $N_{1}=\{0,1,2, \ldots\}$, we use the ceiling function $\lceil\alpha\rceil$ to denote the smallest integer greater than or equal to $\alpha$.

\subsection{Shifted Chebyshev polynomials}

The well-known Chebyshev polynomials can be defined on the interval $x \in[-1,1]$ and can be determined with the following recurrence formula:

$$
\left\{\begin{array}{l}
P_{0}^{*}\left(x^{*}\right)=1, \quad P_{1}^{*}\left(x^{*}\right)=x^{*} \\
P_{i+1}^{*}\left(x^{*}\right)=2 x^{*} P_{i}^{*}\left(x^{*}\right)-P_{i-1}^{*}\left(x^{*}\right), \quad i=1,2,3, \ldots
\end{array}\right.
$$

In order to obtain these polynomials on the interval $[0,1]$, we introduce the change of variable $x^{*}=2 x-1$ and substitute $x^{*}$ to $P_{i}^{*}\left(x^{*}\right), i=0,1,2, \ldots$. The shifted Chebyshev polynomials can be defined as

$$
\left\{\begin{array}{l}
P_{0}(x)=1, \quad P_{1}(x)=2 x-1, \\
P_{i+1}(x)=2(2 x-1) P_{i}(x)-P_{i-1}(x), \quad i=1,2,3, \ldots
\end{array}\right.
$$

The shifted Chebyshev polynomials $P_{n}(x)$ of degree $\mathrm{n}$ can be given by

$$
P_{n}(x)=\sum_{i=0}^{n}(-1)^{n-i} 2^{2 i} \frac{n(n+i-1) !}{(n-i) !(2 i) !} x^{i}
$$

the weight function is

$$
w_{s}(x)=\frac{1}{\sqrt{x-x^{2}}} .
$$

Combining (7) the orthogonality condition is

$$
\int_{0}^{1} P_{n}(x) P_{m}(x) \frac{d x}{\sqrt{x-x^{2}}} \begin{cases}0, & n \neq m \\ \pi, & n=m=0 \\ \pi / 2, & n=m \neq 0\end{cases}
$$


We can define the shifted Chebyshev vector as follows:

$$
\Phi(x)=\left[P_{0}(x), P_{1}(x), \ldots, P_{n}(x)\right]^{T},
$$

the vector is represented as a matrix form as follows:

$$
\Phi(x)=A T_{n}(x)
$$

where

$$
A=\left[\begin{array}{cccc}
1 & 0 & \cdots & 0 \\
4^{0} \frac{1(1+0-1) !}{(1-0) !(0) !} & 4^{1} \frac{1(1+1-1) !}{(1-1) !(2) !} & \cdots & 0 \\
\vdots & \vdots & \ddots & \vdots \\
4^{0} \frac{n(n+0-1) !}{(n-0) !(0) !} & 4^{1 \frac{n(n+1-1) !}{(n-1) !(2) !}} & \cdots & 4^{n} \frac{n(n+n-1) !}{(n-n) !(2 n) !}
\end{array}\right]
$$

\section{Function approximation}

The function $u(x)$ is a continuous function which can be expanded in shifted Chebyshev polynomials:

$$
u(x)=\sum_{i=0}^{\infty} c_{i} P_{i}(x)
$$

A finite expansion in the first $(n+1)$-term shifted Chebyshev polynomials is

$$
u(x) \cong \sum_{i=0}^{n} c_{i} P_{i}(x)=C^{T} \Phi(x),
$$

where the shifted Chebyshev vector $\Phi(x)$ and the shifted Chebyshev coefficient vector $C$ are given by

$$
\begin{aligned}
& C=\left[c_{0}, c_{1}, \ldots, c_{n}\right]^{T}, \\
& \Phi(x)=\left[P_{0}(x), P_{1}(x), \ldots, P_{n}(x)\right]^{T} .
\end{aligned}
$$

The coefficient vector $C$ can be determined by the inner product

$$
C=Q^{-1}\langle u, \Phi(x)\rangle,
$$

where the inner product is defined as

$$
\langle f, \Phi(x)\rangle=\int_{0}^{x_{f}} f(x) \Phi(x) \mathrm{d} x
$$


where $Q$ is

$$
\begin{aligned}
Q & =\int_{0}^{x_{f}} \Phi(x) \Phi^{T}(x) \mathrm{d} x \\
& =\int_{0}^{x_{f}}(A T(x))(A T(x))^{T} \mathrm{~d} x \\
& =A\left(\int_{0}^{x_{f}} T(x) T^{T}(x) \mathrm{d} x\right) A^{T} \\
& =A H A^{T},
\end{aligned}
$$

where $x_{f}=1, H$ is

$$
H=\left[\begin{array}{cccc}
1 & \frac{1}{2} & \cdots & \frac{1}{n+1} \\
\frac{1}{2} & \frac{1}{3} & \cdots & \frac{1}{n+2} \\
\vdots & \vdots & \ddots & \vdots \\
\frac{1}{n+1} & \frac{1}{n+2} & \cdots & \frac{1}{2 n+2}
\end{array}\right]
$$

\section{Error correction and convergence analysis}

In this section, we do error correction for multi-order fractional differential equation and introduce convergence of shifted Chebyshev polynomials. The order of convergence is $n$.

\subsection{Error correction}

We solve multi-order fractional differential equation via the shifted Chebyshev polynomials. If the absolute error between the numerical solution and exact solution is larger, according to the correct solution and the exact solution, we can get the absolute error of correct solution. Error correction improves the precision of numerical solution.

We assume that the numerical solution of multi-order fractional differential equation is $u_{M}(x)$, the exact solution is $u(x)$, the error between the numerical solution and the exact solution is

$$
e_{M}(x)=u(x)-u_{M}(x)
$$

where $e_{M}(x)$ is an error function.

Substituting the numerical solution of equation $u_{M}(x)$ in multi-order fractional differential equation, we can get

$$
D^{\alpha} u_{M}(x) \approx \sum_{i=0}^{k} y_{i} D^{\beta_{i}} u_{M}(x)+f(x) .
$$

A residual function $w_{M}(x)$ is added to the right-hand side of the multi-order fractional differential equation, (13) can be transformed into

$$
D^{\alpha} u_{M}(x)=\sum_{i=0}^{k} y_{i} D^{\beta_{i}} u_{M}(x)+f(x)+w_{M}(x) .
$$


Then we can get the equation

$$
Q\left[u_{M}(x)\right]=f(x)+w_{M}(x)
$$

where

$$
Q\left[u_{M}(x)\right]=D^{\alpha} u_{M}(x)-\sum_{i=0}^{k} y_{i} D^{\beta_{i}} u_{M}(x) .
$$

We assume that $\phi$ is the unknown variables in the following equation:

$$
Q[\phi]=D^{\alpha} \phi-\sum_{i=0}^{k} y_{i} D^{\beta_{i}} \phi,
$$

when $\phi=u(x)$ and $\phi=e_{M}(x)$, we have

$$
\begin{aligned}
& Q[u(x)]=D^{\alpha} u(x)-\sum_{i=0}^{k} y_{i} D^{\beta_{i}} u(x)=f(x), \\
& Q\left[e_{M}(x)\right]=D^{\alpha} e_{M}(x)-\sum_{i=0}^{k} y_{i} D^{\beta_{i}} e_{M}(x) .
\end{aligned}
$$

Combining (12) and (15)-(19), we can obtain

$$
Q\left[e_{M}(x)\right]=Q[u(x)]-Q\left[u_{M}(x)\right]=-w_{M}(x)
$$

According to (19) and (20), we can get

$$
D^{\alpha} e_{M}(x)-\sum_{i=0}^{k} y_{i} \mathrm{D}^{\beta_{i}} e_{M}(x)=-w_{M}(x) .
$$

We name (21) multi-order fractional differential equation of error function. $e_{M}(x)$ is the exact solution, $e_{\omega}^{*}(x)$ is the numerical solution, namely the approximate error function.

According to the numerical solution of multi-order fractional differential equation $u_{M}(x)$ and the numerical solution of multi-order fractional differential equation of error function $e_{\omega}^{*}(x)$, correct solution $u^{*}(x)$ can be obtained:

$$
u^{*}(x)=u_{M}(x)+e_{\omega}^{*}(x)
$$

Combining (22) with the exact solution $u(x)$, we can get the absolute error of correct solution:

$$
\left|e_{r}(x)\right|=\left|u(x)-u^{*}(x)\right|
$$

The error $e_{r}(x)$ between the exact solution and the numerical solution of multi-order fractional differential equation of error function is

$$
e_{r}(x)=e_{M}(x)-e_{\omega}^{*}(x)=u(x)-u_{M}(x)-e_{\omega}^{*}(x)
$$

$e_{r}(x)$ is a correction error function. 
In the same way, according to the shifted Chebyshev polynomials function approximation theory, correct solution $u^{*}(x)$ can be translated into a matrix form as follows:

$$
u^{*}(x) \cong \sum_{i=0}^{m} c_{i}^{*} P_{i}(x)=\left(C^{*}\right)^{T} \Phi^{*}(x),
$$

where $\left(n<m \in N^{*}\right)$

$$
\begin{aligned}
& C^{*}=\left[c_{0}^{*}, c_{1}^{*}, \ldots C_{m}^{*}\right]^{T}, \\
& \Phi^{*}(x)=\left[P_{0}(x), P_{1}(x), \ldots, P_{m}(x)\right]^{T} .
\end{aligned}
$$

\subsection{Convergence analysis}

Definition 2 In the interval $[a, b]$, we can define arbitrary function convergence coefficient of form as follows:

$$
\omega(f, \delta)=\sup _{x, y \in[a, b],|x-y| \leq \delta}|f(x)-f(y)| .
$$

Theorem 1 In $[a, b]$, the function is uniformly convergent if and only if $\lim _{\delta \rightarrow 0} \omega(f, \delta)=0$.

Theorem 2 If $f(x)$ is bounded on $[0,1]$, then there is

$$
\|f-q(f, n)\|_{\infty} \leq \frac{3}{2} \omega\left(f, \frac{1}{\sqrt{n}}\right)
$$

where

$$
q(f, n)=\sum_{k=0}^{n} f\left(\frac{k}{n}\right) P_{k},
$$

and

$$
\|f\|_{\infty}=\sup |f(x)| .
$$

Theorem 3 When $f(x)$ satisfies $\alpha$ order Lipschitz condition in $[0,1]$, then there is

$$
\|f-q(f, n)\|_{\infty} \leq \frac{3}{2} k m^{-\frac{\alpha}{2}}
$$

where $k$ is a Lipschitz constant.

Theorem 4 If $f(x)$ is bounded on $[0,1], Y=\operatorname{Span}\left\{P_{0}, P_{1}, P_{2}, \ldots, P_{n}\right\}$. If $C^{T} \Phi(x)$ is the best approximation of $f$ in the linear space $Y$, then there is

$$
\left\|f-c^{T} \Phi\right\|_{2} \leq \frac{3}{2} \omega\left(f, \frac{1}{\sqrt{n}}\right) .
$$

It is confirmed that shifted Chebyshev polynomials approximation converges to $f$; when $n$ converges to $\infty$, approximation consistently converges to $f$. 


\section{Fractional differential operator matrix}

According to (10), the differential operator can be derived as follows:

$$
\begin{aligned}
\mathrm{D} \Phi(x) & =D\left(A T_{n}(x)\right)=A \mathrm{D}\left(T_{n}(x)\right) \\
& =A \mathrm{D}\left[\begin{array}{c}
1 \\
x \\
\vdots \\
x^{n}
\end{array}\right]=A \mathrm{D}\left[\begin{array}{c}
0 \\
1 \\
\vdots \\
x^{n-1}
\end{array}\right]=A Q_{(n+1) \times n} T_{n}^{*}(x) .
\end{aligned}
$$

The above formula can be shown specifically as follows:

$$
\begin{aligned}
& Q_{(n+1) \times n}=\left[\begin{array}{cccc}
0 & 0 & 0 & 0 \\
1 & 0 & \cdots & 0 \\
0 & 2 & \cdots & 0 \\
\vdots & \vdots & \ddots & \vdots \\
0 & 0 & \cdots & n
\end{array}\right], \\
& T_{n}^{*}(x)=B^{*} \Phi(x), \\
& B^{*}=\left[A_{[1]}^{-1}, A_{[2]}^{-1}, \ldots, A_{[\mathrm{n}]}^{-1}\right]^{T},
\end{aligned}
$$

where $A_{[\eta]}^{-1}$ is line $\eta$ of $A^{-1}, \eta=1,2, \ldots, n$.

So

$$
\mathrm{D} \Phi(x)=D\left(A T_{n}(x)\right)=A Q_{(n+1) \times n} T_{n}^{*}(x)=E \Phi(x) .
$$

First-order differential operator matrix is

$$
E=A Q_{(n+1) \times n} B^{*} .
$$

When $\beta_{2} \in[0,1)$, combining (3) with (10), we can deduce

$$
\begin{aligned}
\mathrm{D}^{\beta_{2}} \Phi(x) & =\mathrm{D}^{\beta_{2}}\left(A T_{n}(x)\right)=A \mathrm{D}^{\beta_{2}} T_{n}(x) \\
& =A \mathrm{D}^{\beta_{2}}\left[\begin{array}{c}
1 \\
x \\
\vdots \\
x^{n}
\end{array}\right]=A\left[\begin{array}{c}
0 \\
\frac{\Gamma(2)}{\Gamma\left(2-\beta_{2}\right)} x^{-\beta_{2}} \\
\vdots \\
\frac{\Gamma(n+1)}{\Gamma\left(n+1-\beta_{2}\right)} x^{-\beta_{2}}
\end{array}\right] \\
& =A\left[\begin{array}{ccccc}
0 & 0 & 0 & \ldots & 0 \\
0 & \frac{\Gamma(2)}{\Gamma\left(2-\beta_{2}\right)} x^{-\beta_{2}} & 0 & \ldots & 0 \\
0 & 0 & \frac{\Gamma(3)}{\Gamma\left(3-\beta_{2}\right)} x^{-\beta_{2}} & \ldots & 0 \\
\vdots & \vdots & \vdots & \ddots & \vdots \\
0 & 0 & 0 & 0 & \frac{\Gamma(n+1)}{\Gamma\left(n+1-\beta_{2}\right)} x^{-\beta_{2}}
\end{array}\right]\left[\begin{array}{c}
1 \\
x \\
\vdots \\
x^{n}
\end{array}\right] \\
& =A N A^{-1} \Phi(x),
\end{aligned}
$$


where

$$
N=\left[\begin{array}{ccccc}
0 & 0 & 0 & \cdots & 0 \\
0 & \frac{\Gamma(2)}{\Gamma\left(2-\beta_{2}\right)} x^{-\beta_{2}} & 0 & \cdots & 0 \\
0 & 0 & \frac{\Gamma(3)}{\Gamma\left(3-\beta_{2}\right)} x^{-\beta_{2}} & \ldots & 0 \\
\vdots & \vdots & \vdots & \ddots & \vdots \\
0 & 0 & 0 & 0 & \frac{\Gamma(n+1)}{\Gamma\left(n+1-\beta_{2}\right)} x^{-\beta_{2}}
\end{array}\right] .
$$

So the fractional differential operator can be deduced

$$
\mathrm{D}^{\beta_{2}} \Phi(x)=A N A^{-1} \Phi(x)=G \Phi(x) .
$$

Fractional differential operator matrix is

$$
G=A N A^{-1} \text {. }
$$

\section{The numerical algorithm}

The multi-order fractional differential equation, which we study in this paper, can be expressed as follows:

$$
\begin{aligned}
D^{\alpha} u(x)= & y_{0} \mathrm{D}^{\beta_{0}} u(x)+y_{1} \mathrm{D}^{\beta_{1}} u(x)+y_{2} \mathrm{D}^{\beta_{2}} u(x) \\
& +y_{3} \mathrm{D}^{\beta_{3}} u(x)+f(x), \quad x \in[0,1]
\end{aligned}
$$

with the initial conditions

$$
u^{(0)}(0)=d_{0}, \quad u^{(1)}(0)=d_{1},
$$

where $\alpha=2, k=3$, the coefficient $y_{i}(i=0,1,2,3)$ is constant, and $\beta_{0}=0, \beta_{1}=1,0<\beta_{2}<1$, $1<\beta_{3}<2, f(x)$ is a known function.

On the basis of (10), (11), (26), the item in equation can be converted into the matrix, we can deduce concretely them as follows:

$$
\begin{aligned}
& \mathrm{D} u(x) \cong \mathrm{D}^{T} \Phi(x)=C^{T} A Q_{(n+1) \times n} B^{*} \Phi(x)=C^{T} E \Phi(x), \\
& \mathrm{D}^{2} u(x) \cong \mathrm{D}^{2} C^{T} \Phi(x)=C^{T} \mathrm{D}^{2} \Phi(x)=C^{T} \mathrm{D}^{2}\left(A T_{n}(x)\right)=C^{T} E^{2} \Phi(x) .
\end{aligned}
$$

Then, the second-order differential operator matrix is

$$
E^{2}=\left(A Q_{(n+1) \times n} B^{*}\right)^{2} .
$$

When $\beta_{2} \in(0,1)$, on the basis of (10), (11), (28), the item in equation can be converted into the matrix

$$
\mathrm{D}^{\beta_{2}} u(x) \cong \mathrm{D}^{\beta_{2}} C^{T} \Phi(x)=C^{T} \mathrm{D}^{\beta_{2}} \Phi(x)=C^{T} A N A^{-1} \Phi(x)=C^{T} G \Phi(x) .
$$


When $\beta_{3} \in(1,2),\left(\beta_{2}<\beta_{3}\right)$, that is to say $\beta_{2}=\beta_{3}-1 \in(0,1)$, according to (10), (11), (26), (28), the item in equation can be converted into the matrix as follows:

$$
\begin{aligned}
\mathrm{D}^{\beta_{3}} u(x) & \cong \mathrm{D}^{\beta_{3}} C^{T} \Phi(x)=\mathrm{D}^{\beta_{2}}\left(D C^{T} \Phi(x)\right)=C^{T} \mathrm{D}^{\beta_{2}}(E \Phi(x)) \\
& =C^{T} E \mathrm{D}^{\beta_{2}} \Phi(x)=C^{T} E A N A^{-1} \Phi(x)=C^{T} E G \Phi(x),
\end{aligned}
$$

where

$$
K=E A N A^{-1}=E G
$$

Combining (11) with (32)-(36), the equation can be converted into

$$
C^{T} E^{2} \Phi(x)=y_{0} C^{T} \Phi(x)+y_{1} C^{T} E \Phi(x)+y_{2} C^{T} G \Phi(x)+y_{3} C^{T} K \Phi(x)+f(x) .
$$

Also, we substitute the correction solution into the original equation and translate the original equation into matrix as follows:

$$
\begin{aligned}
\left(C^{*}\right)^{T} E^{2} \Phi^{*}(x)= & y_{0}\left(C^{*}\right)^{T} \Phi^{*}(x)+y_{1}\left(C^{*}\right)^{T} E \Phi^{*}(x) \\
& +y_{2}\left(C^{*}\right)^{T} G \Phi^{*}(x)+y_{3}\left(C^{*}\right)^{T} K \Phi^{*}(x)+f(x) .
\end{aligned}
$$

By using the collocation method, the variables are discretized, the problem can be transferred to linear equations. Combining MATLAB software with least square method to solve the unknown coefficient, numerical solution of the problem can be obtained.

\section{Numerical examples}

In this section, two experiments prove that the proposed method is effective and feasible.

Example 1 Consider the following multi-order fractional differential equation:

$$
\begin{aligned}
D^{\alpha} u(x)= & y_{0} \mathrm{D}^{\beta_{0}} u(x)+y_{1} \mathrm{D}^{\beta_{1}} u(x)+y_{2} \mathrm{D}^{\beta_{2}} u(x) \\
& +y_{3} \mathrm{D}^{\beta_{3}} u(x)+f(x), \quad x \in[0,1]
\end{aligned}
$$

with the initial conditions

$$
u^{(0)}(0)=d_{0}, u^{(1)}(0)=d_{1},
$$

where $\alpha=2, d_{0}=d_{1}=0$, the coefficient is $y_{0}=y_{2}=-1, y_{1}=2, y_{3}=0$, and $\beta_{0}=0, \beta_{1}=1$, $\beta_{2}=\frac{1}{2} \in(0,1)$, the known function is

$$
f(x)=x^{7}+\frac{2048}{429 \sqrt{\pi}} x^{6.5}-14 x^{6}+42 x^{5}-x^{2}-\frac{8}{3 \sqrt{\pi}} x^{1.5}+4 x-2,
$$

the exact solution is $u(x)=x^{7}-x^{2}$.

When $n=4$, the discrete variable is $x_{i}=\frac{i}{5}-\frac{1}{10}(i=1,2, \ldots, 5)$, the numerical solution is $u(x)=C_{1}^{T} \Phi(x)$, the unknown coefficient can be obtained $C_{1}=[0.3602,0.8710,0.1714$, 
$0.1750,0.0924]^{T}$, the shifted Chebyshev polynomials of approximation function $\Phi(x)$ are

$$
\Phi(x)=\left[\begin{array}{c}
1 \\
2 x-1 \\
8 x^{2}-8 x+1 \\
32 x^{3}-48 x^{2}+18 x-1 \\
128 x^{4}-256 x^{3}+160 x^{2}-32 x+1
\end{array}\right] .
$$

When $n=6$, the discrete variable is $x_{i}=\frac{i}{7}-\frac{1}{14}(i=1,2, \ldots, 7)$, the numerical solution is $u(x)=C_{1}^{T} \Phi(x)$, the unknown coefficient can be obtained $C_{1}=[-0.1528,-0.1193,0.1207$, $0.1220,0.0441,0.0113,0.0020]^{T}$, the shifted Chebyshev polynomials of approximation function $\Phi(x)$ are

$$
\Phi(x)=\left[\begin{array}{c}
1 \\
2 x-1 \\
8 x^{2}-8 x+1 \\
32 x^{3}-48 x^{2}+18 x-1 \\
128 x^{4}-256 x^{3}+160 x^{2}-32 x+1 \\
512 x^{5}-1280 x^{4}+1120 x^{3}-400 x^{2}+50 x-1 \\
2048 x^{6}-6144 x^{5}+6912 x^{4}-3584 x^{3}+840 x^{2}-72 x+1
\end{array}\right] .
$$

When $n=7$, the discrete variable is $x_{i}=\frac{i}{8}-\frac{1}{16}(i=1,2, \ldots, 8)$, the numerical solution is $u(x)=C_{1}^{T} \Phi(x)$, the unknown coefficient can be obtained $C_{1}=[-0.1655,-0.1334,0.1194$, $0.1222,0.0444,0.0111,0.0017,0.0001]^{T}$, the shifted Chebyshev polynomials of approximation function $\Phi(x)$ are

$$
\Phi(x)=\left[\begin{array}{c}
1 \\
2 x-1 \\
8 x^{2}-8 x+1 \\
32 x^{3}-48 x^{2}+18 x-1 \\
128 x^{4}-256 x^{3}+160 x^{2}-32 x+1 \\
512 x^{5}-1280 x^{4}+1120 x^{3}-400 x^{2}+50 x-1 \\
2048 x^{6}-6144 x^{5}+6912 x^{4}-3584 x^{3}+840 x^{2}-72 x+1 \\
8192 x^{7}-28672 x^{6}+39424 x^{5}-26880 x^{4}+9408 x^{3}-1568 x^{2}+98 x-1
\end{array}\right] .
$$

Compared with [30], Example 1 studies the approximation effect of numerical solution and exact solution, the absolute errors and the absolute error of correct solution. When $n=4,6,7$, the absolute errors for equation in some match points between the numerical solution and the exact solution are shown in Fig. 1 -Fig. 3 and Table 1. When $n=6,7,8$, the numerical solution and exact solution are shown in Fig. 4-Fig. 6.

When $n=4$, the absolute error is bigger, we do correction for the numerical solution with $n=4$ and obtain the correct solution with $n=4, m=8$, the correct solution and the absolute error of correct solution are shown in Fig. 7 and Fig. 8.

From Fig. 1-Fig. 3 and Table 1, we see that, when $n=4,6$, the absolute error is bigger, when $n=7$, the absolute error becomes smaller and the precision of numerical solution is higher, the absolute error achieves about $10^{-15}$. According to Fig. 4-Fig. 6, as the order 


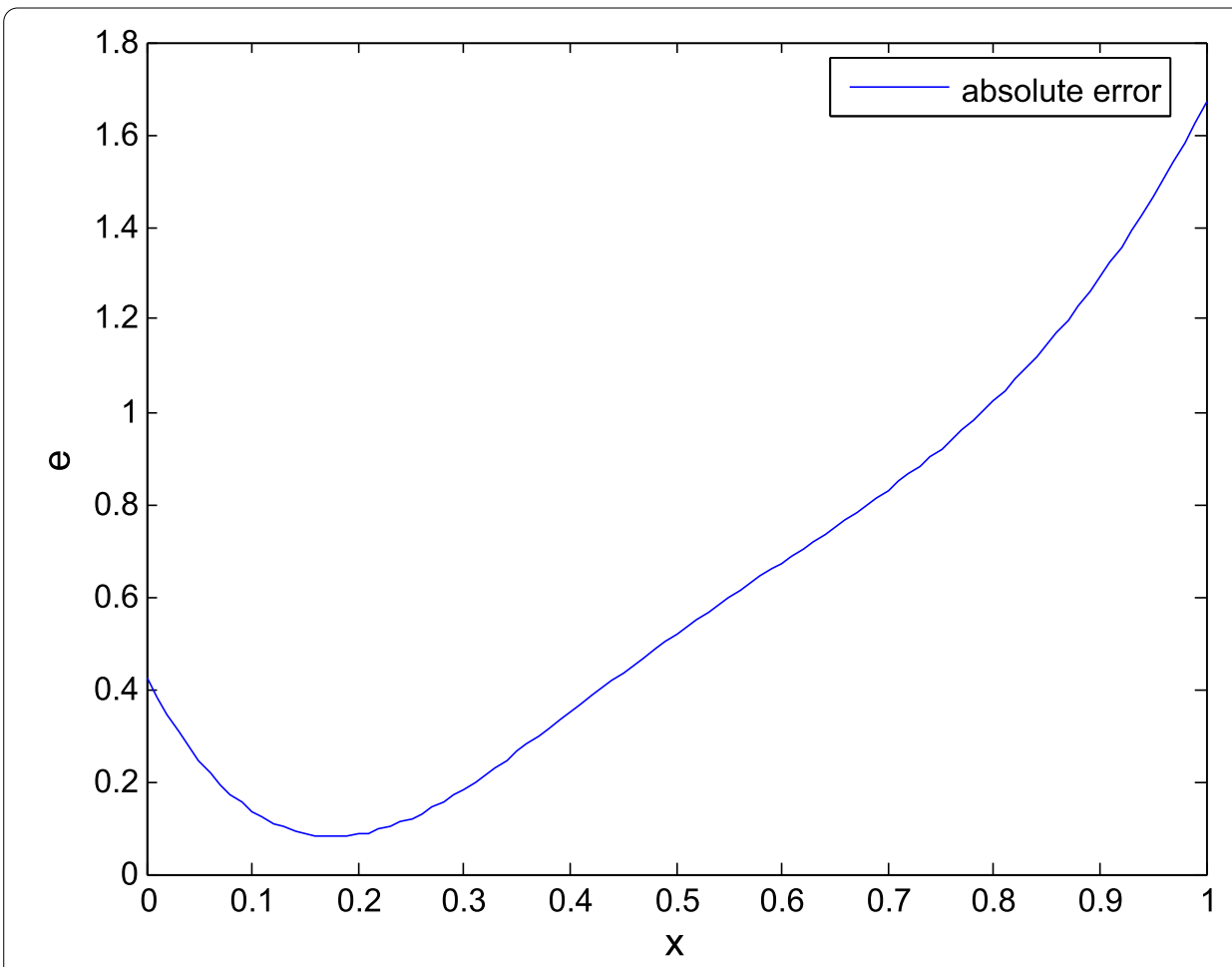

Figure 1 The absolute error between the numerical solution and the exact solution with $n=4$ for Example 1

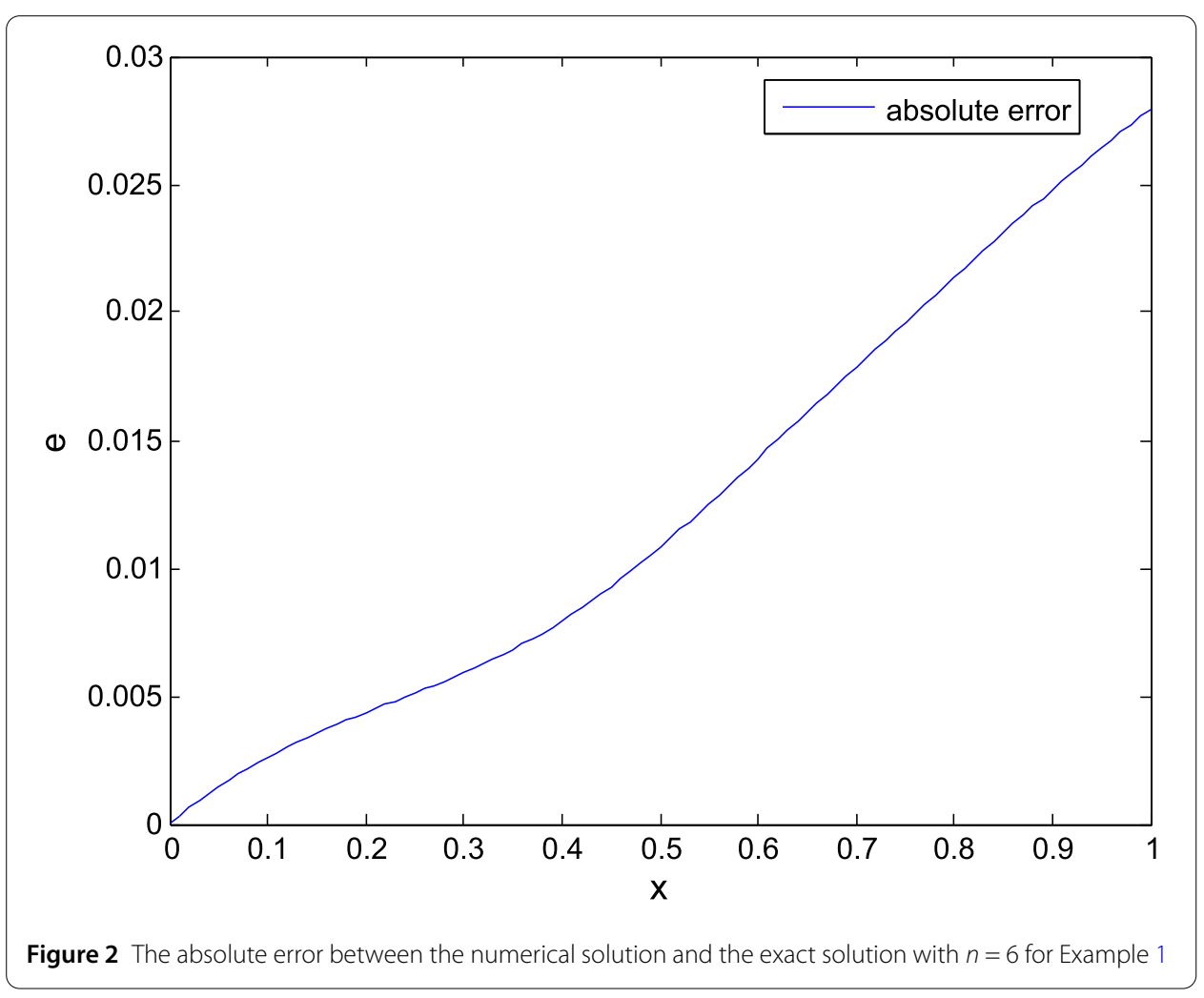




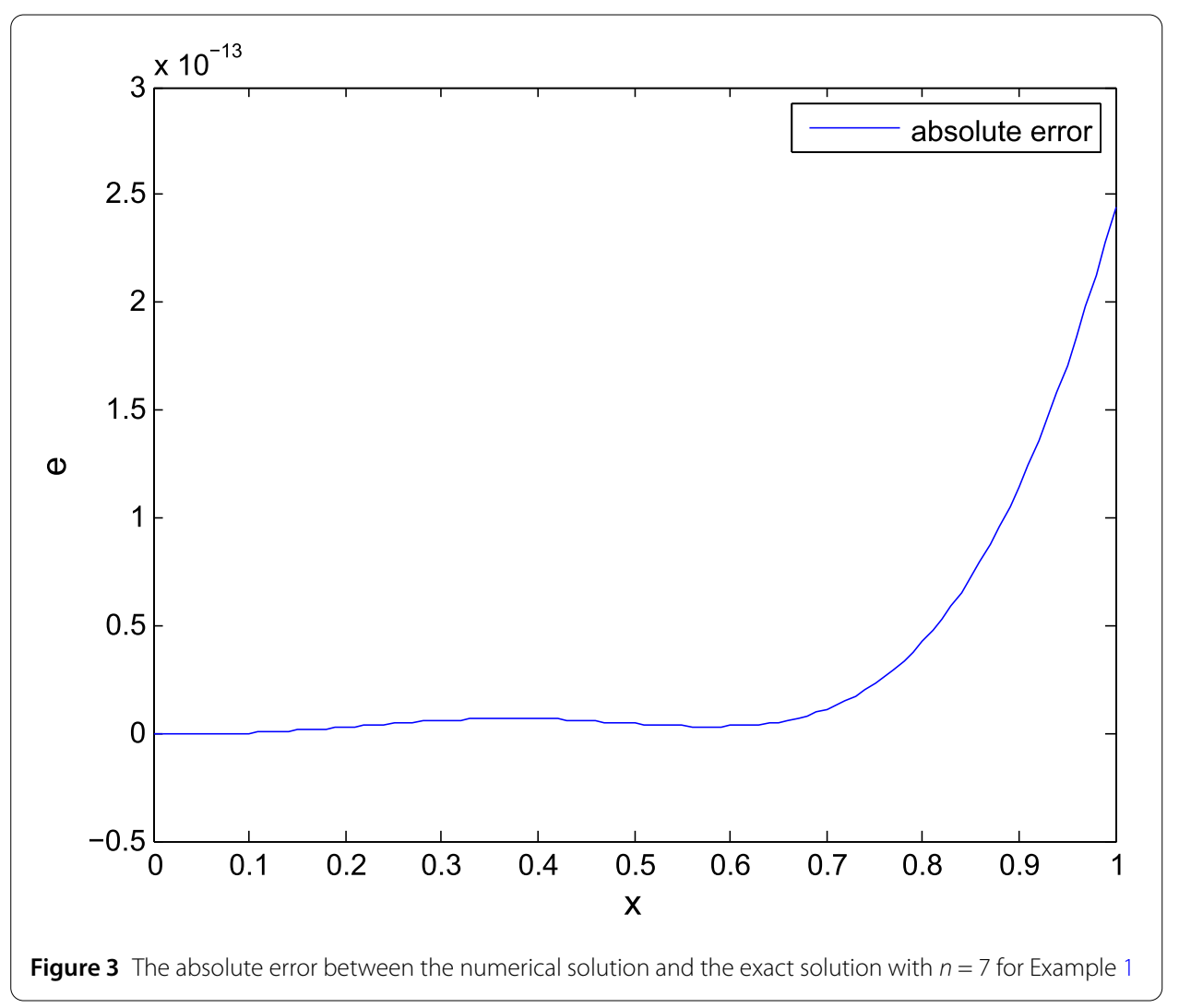

Table 1 The absolute errors with $n=4,6,7$ for Example 1

\begin{tabular}{llll}
\hline$x$ & The absolute errors with $n=4$ & The absolute errors with $n=6$ & The absolute errors with $n=7$ \\
\hline 0.2 & 0.0844 & 0.0044 & $2.81025203108243 \mathrm{e}-15$ \\
0.4 & 0.3501 & 0.0079 & $6.63358257213531 \mathrm{e}-15$ \\
0.6 & 0.6734 & 0.0143 & $3.27515792264421 \mathrm{e}-15$ \\
0.8 & 1.0234 & 0.0214 & $4.25770529943748 \mathrm{e}-14$ \\
1 & 1.6700 & 0.0280 & $2.43819897540083 \mathrm{e}-13$ \\
\hline
\end{tabular}

of convergence $n$ gets larger, the approximation between the numerical solution and the exact solution is better.

From Fig. 1, Fig. 7, Fig. 8, we see that the absolute error of correct solution is smaller than the absolute error of numerical solution.

Example 2 Consider the following multi-order fractional differential equation:

$$
\begin{aligned}
D^{\alpha} u(x)= & y_{0} \mathrm{D}^{\beta_{0}} u(x)+y_{1} \mathrm{D}^{\beta_{1}} u(x)+y_{2} \mathrm{D}^{\beta_{2}} u(x) \\
& +y_{3} \mathrm{D}^{\beta_{3}} u(x)+f(x), \quad x \in[0,1],
\end{aligned}
$$

with the initial conditions

$$
u^{(0)}(0)=d_{0}, \quad u^{(1)}(0)=d_{1},
$$




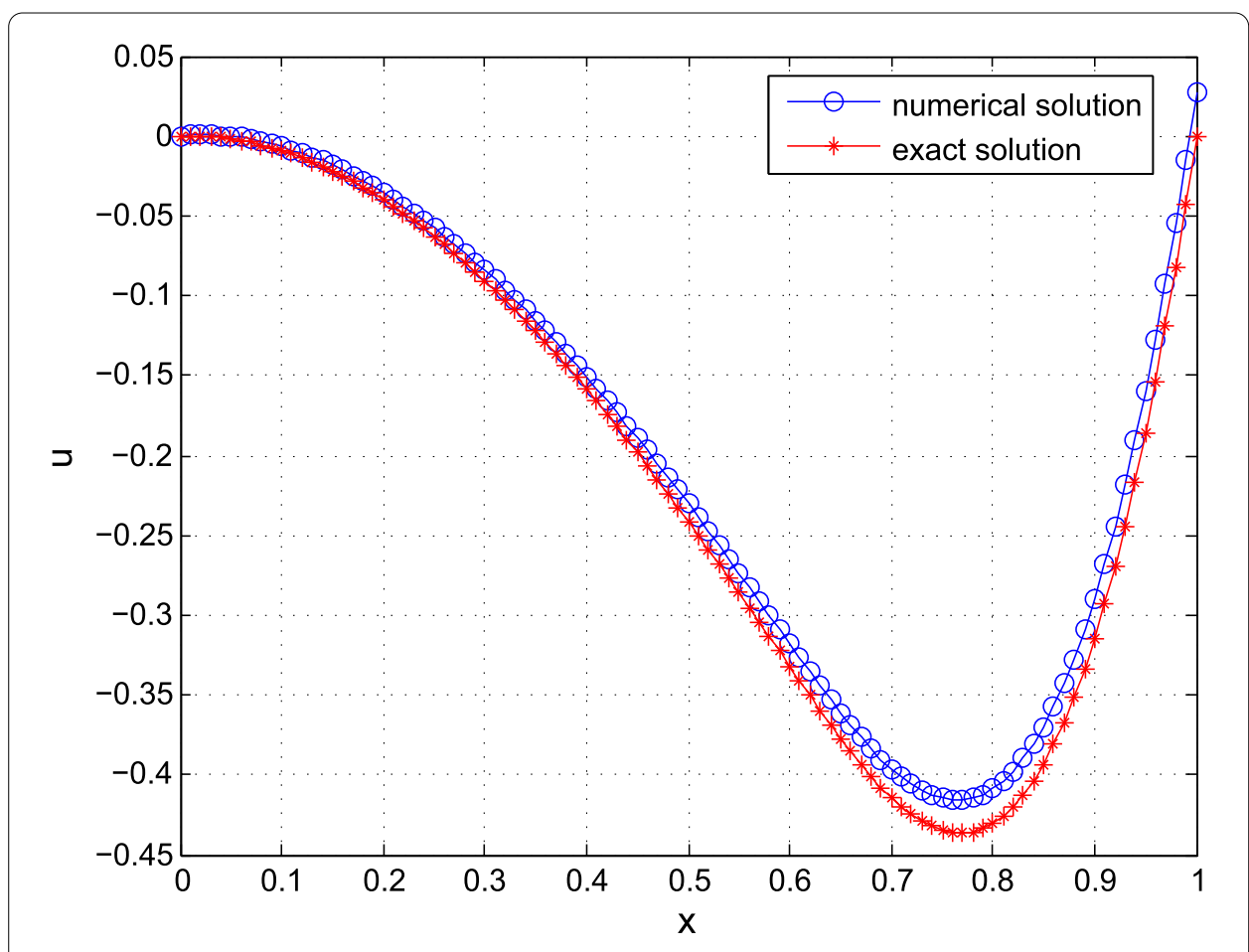

Figure 4 The numerical solution and the exact solution with $n=6$ for Example 1

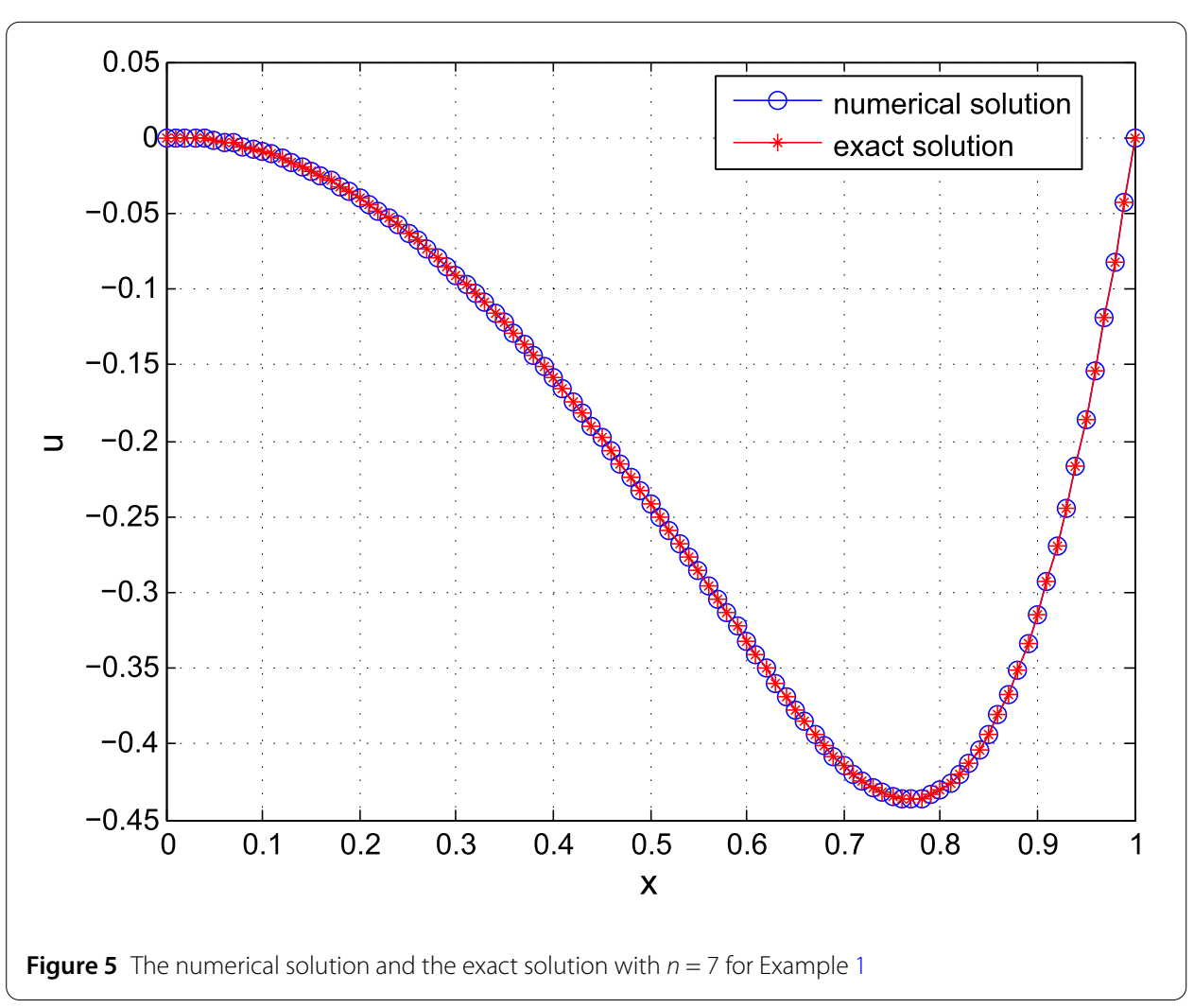




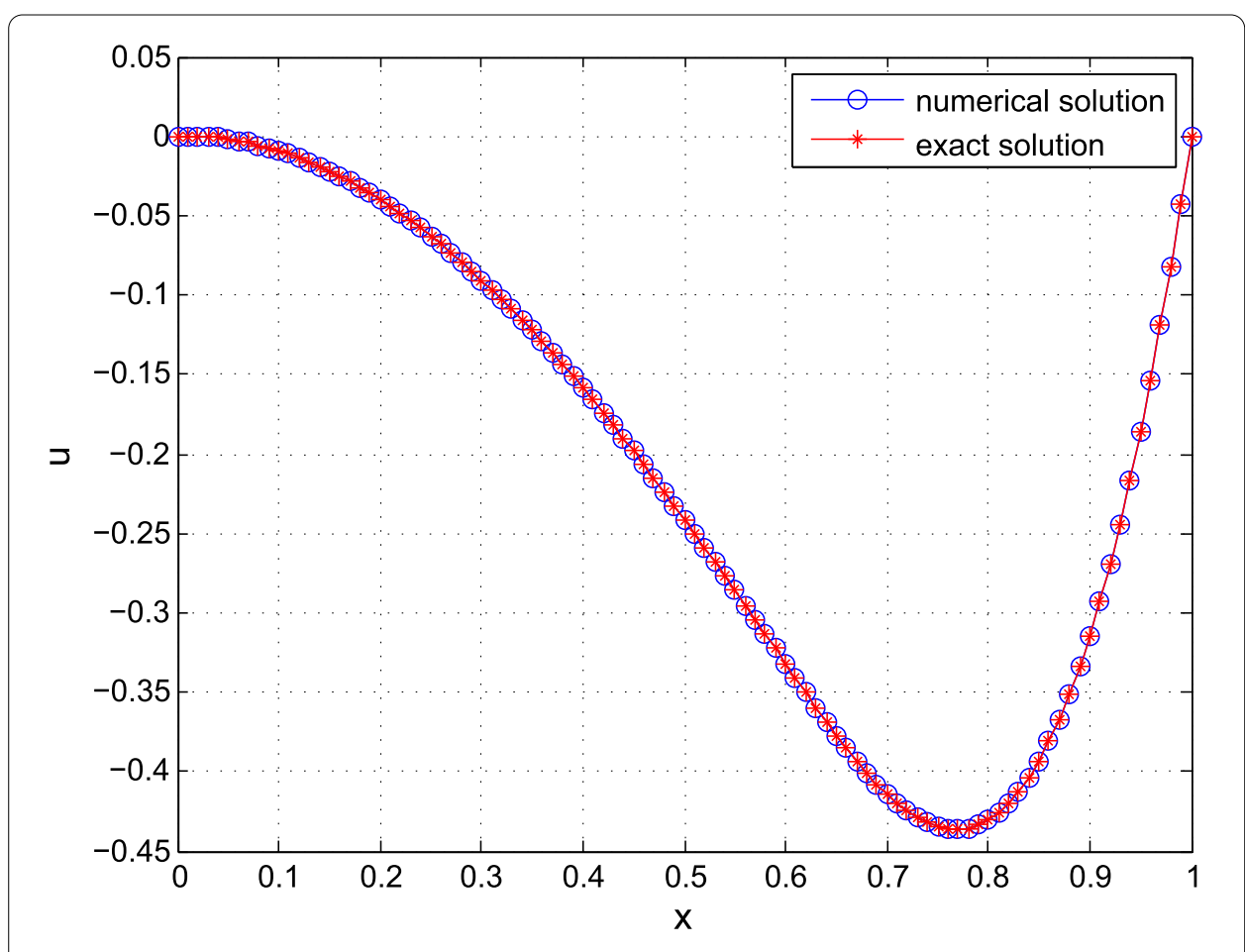

Figure 6 The numerical solution and the exact solution with $n=8$ for Example 1

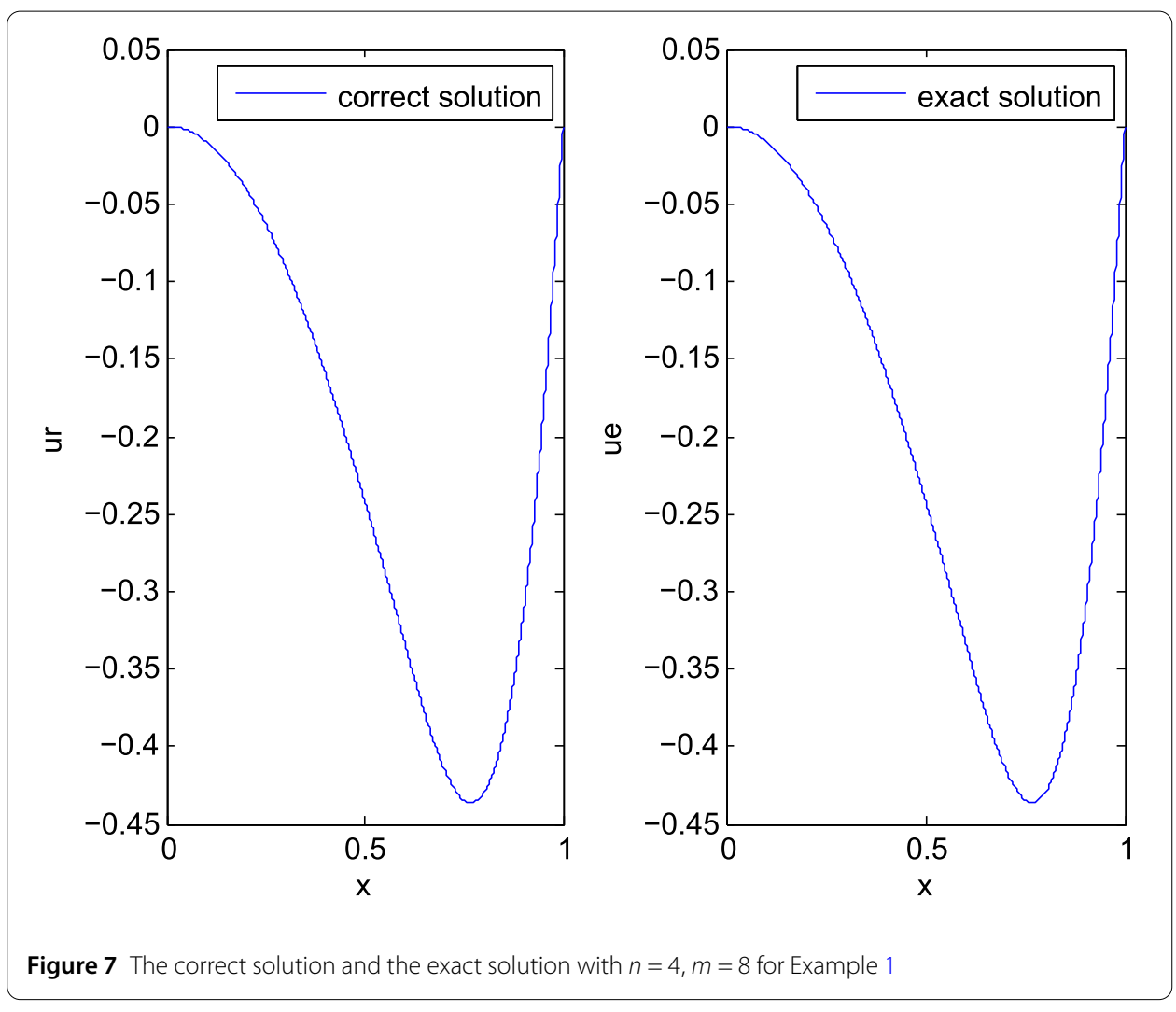




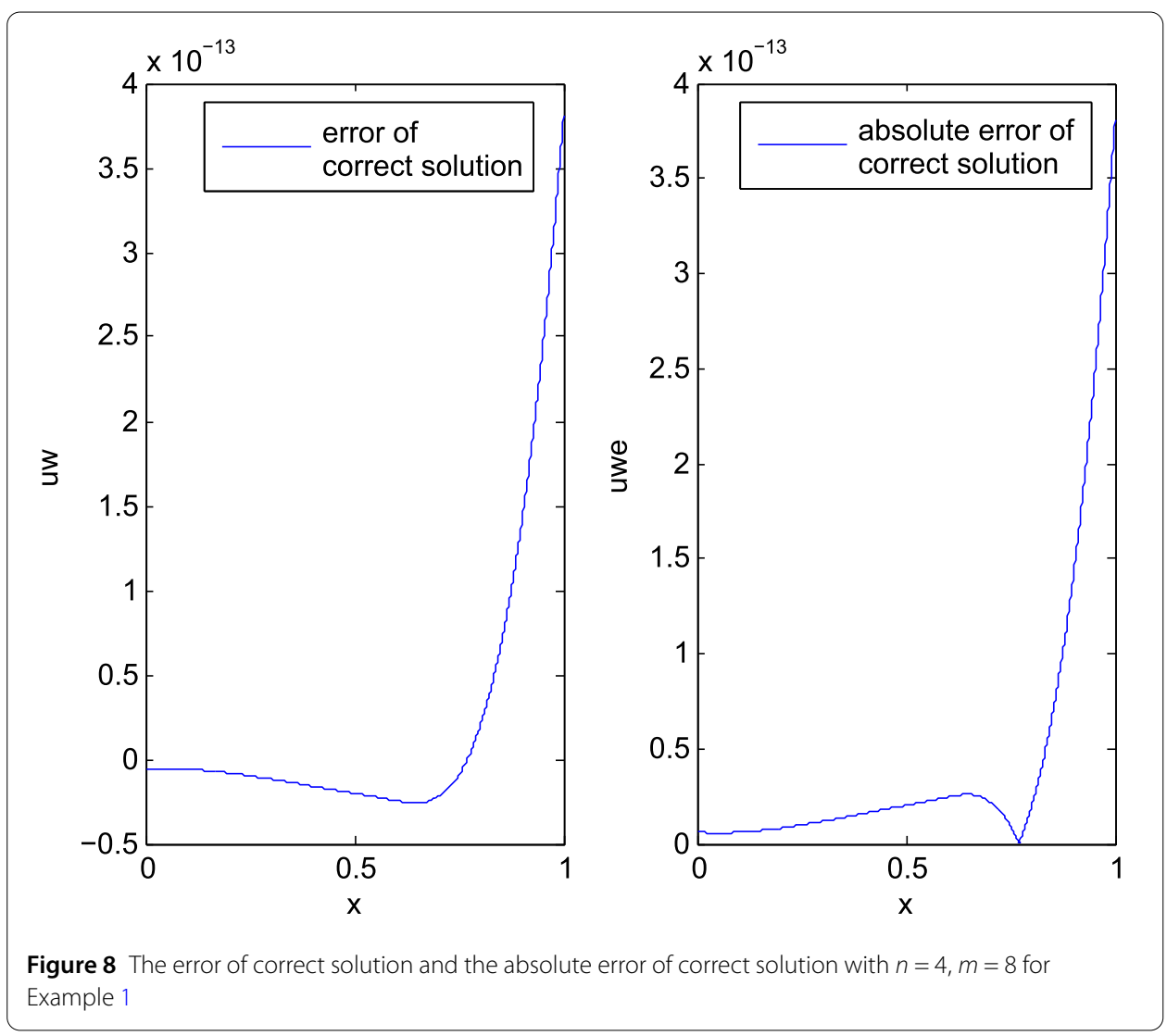

where $\alpha=2, d_{0}=d_{1}=0$, the coefficient is $y_{0}=y_{2}=-1, y_{1}=0, y_{3}=2$, and $\beta_{0}=0, \beta_{2}=\frac{2}{3} \in$ $(0,1), \beta_{3}=\frac{5}{3} \in(1,2)$. The known function is

$$
f(x)=x^{3}+6 x-\frac{12}{\Gamma\left(\frac{7}{3}\right)} x^{\frac{4}{3}}+\frac{6}{\Gamma\left(\frac{10}{3}\right)} x^{\frac{7}{3}},
$$

the exact solution is $u(x)=x^{3}$.

When $n=2$, the discrete variable is $x_{i}=\frac{i}{3}-\frac{1}{6}(i=1,2,3)$, the numerical solution is $u(x)=$ $C_{1}^{T} \Phi(x)$, the unknown coefficient can be obtained $C_{1}=[-0.0912,-0.0695,0.0139]^{T}$, the shifted Chebyshev polynomials of approximation function $\Phi(x)$ are

$$
\Phi(x)=\left[\begin{array}{c}
1 \\
2 x-1 \\
8 x^{2}-2 x-1
\end{array}\right] .
$$

When $n=3$, the discrete variable is $x_{i}=\frac{i}{4}-\frac{1}{8}(i=1,2,3,4)$, the numerical solution is $u(x)=C_{1}^{T} \Phi(x)$, the unknown coefficient can be obtained $C_{1}=[0.3125,0.4688,0.1875$, $0.0313]^{T}$, the shifted Chebyshev polynomials of approximation function $\Phi(x)$ are

$$
\Phi(x)=\left[\begin{array}{c}
1 \\
2 x-1 \\
8 x^{2}-2 x-1 \\
32 x^{3}-48 x^{2}+18 x-1
\end{array}\right]
$$


When $n=2,3$, the absolute errors for equation in some match points between the numerical solution and the exact solution are shown in Fig. 9, Fig. 10, and Table 2. When $n=3,4$, the numerical solution and the exact solution are shown in Fig. 11, Fig. 12.
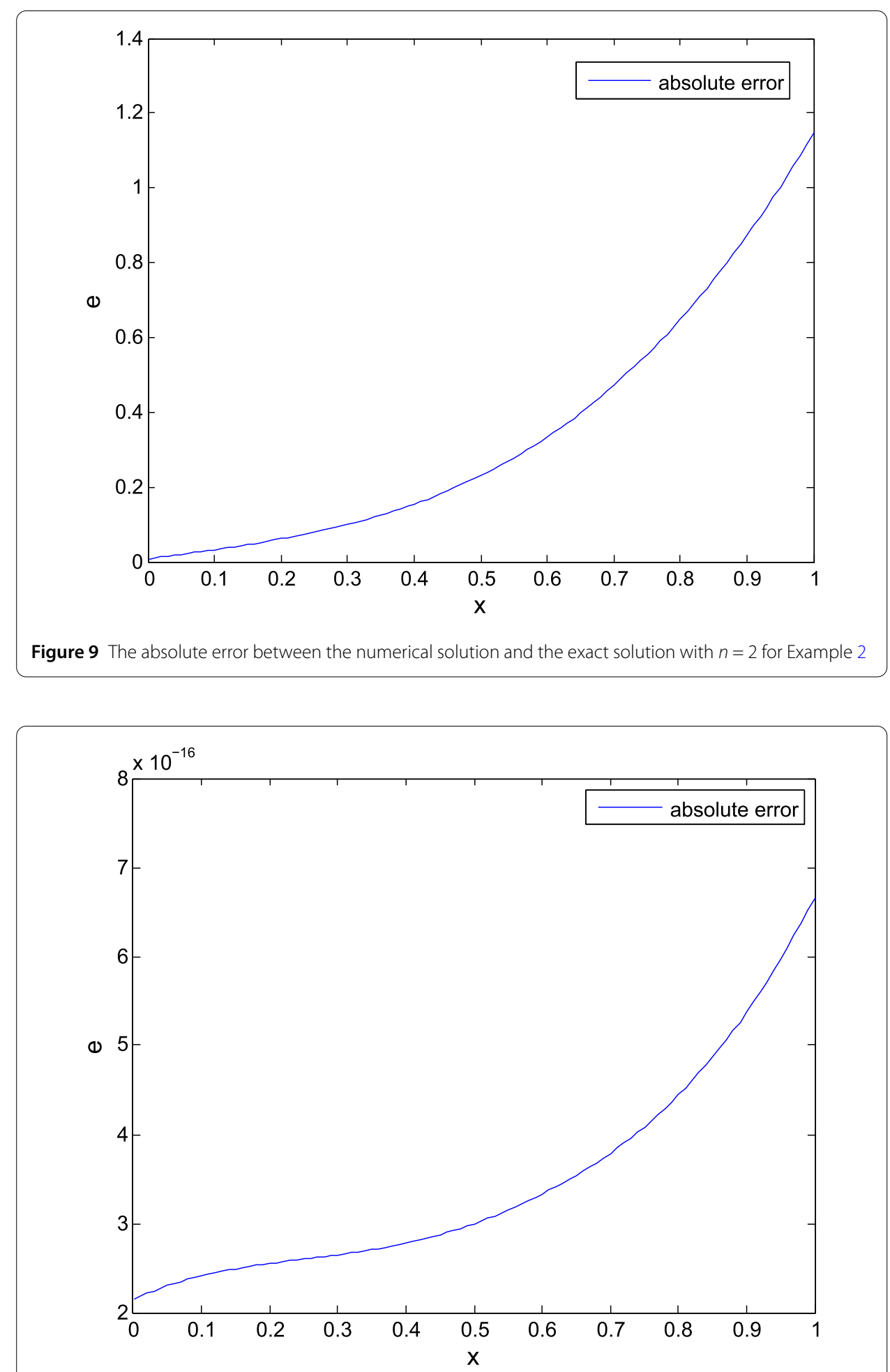

Figure 10 The absolute error between the numerical solution and the exact solution with $n=3$ for Example 2 
Table 2 The absolute errors with $n=2,3$ for Example 2

\begin{tabular}{llll}
\hline$x$ & The exact solution & The absolute errors with $n=2$ & The absolute errors with $n=3$ \\
\hline 0.2 & 0.0080 & 0.0614 & $2.55004350968591 \mathrm{e}-16$ \\
0.4 & 0.0640 & 0.1541 & $2.77555756156289 \mathrm{e}-16$ \\
0.6 & 0.2160 & 0.3339 & $3.33066907387547 \mathrm{e}-16$ \\
0.8 & 0.5120 & 0.6488 & $4.44089209850063 \mathrm{e}-16$ \\
1 & 1.0000 & 1.1468 & $6.66133814775094 \mathrm{e}-16$ \\
\hline
\end{tabular}

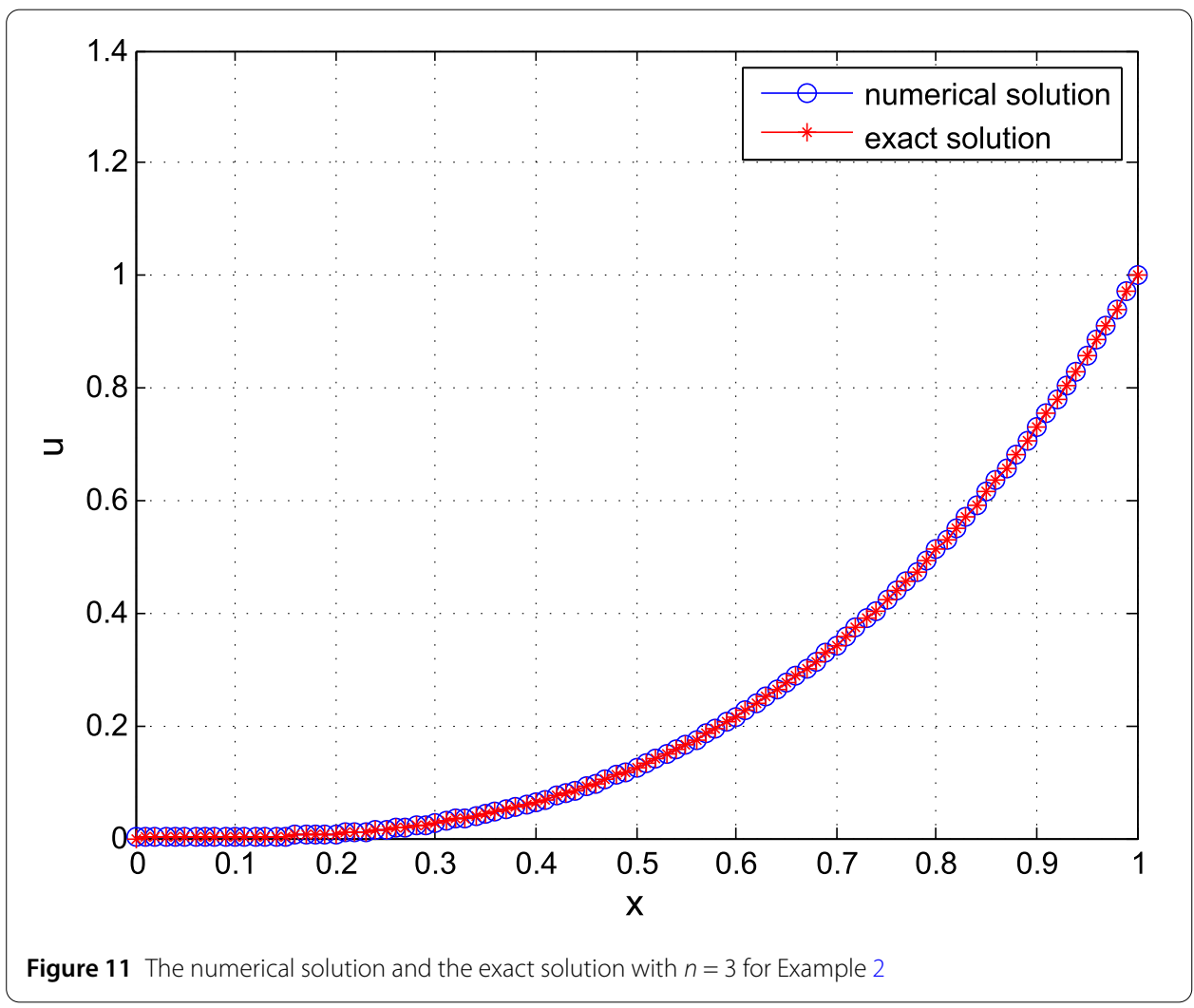

When $n=2$, the absolute error is bigger, we do the correction for the numerical solution with $n=2$ and obtain the correct solution with $n=2, m=4$, the correct solution and the absolute error of correct solution are shown in Fig. 13, Fig. 14.

The author of [32] researched the exact solution of Example 2 to obtain the absolute error of the correction solution, so that the accuracy of the numerical solution is improved. From Fig. 9, Fig. 10, and Table 2, we see that, when $n=2$, the absolute error is bigger, when $n=3$, the absolute error becomes smaller and the precision of numerical solution is higher, the absolute error achieves about $10^{-16}$. From Fig. 11, Fig. 12, we see that, as the order of convergence $n$ gets larger, the convergence effect between the numerical solution and the exact solution is better.

From Fig. 9, Fig. 13, Fig. 14, it is seen that the absolute error of correct solution is clearly smaller.

\section{Conclusion}

In this paper, we conclude that the method based on shifted Chebyshev polynomials is a suitable technique for solving multi-order fractional differential equation. Not only frac- 


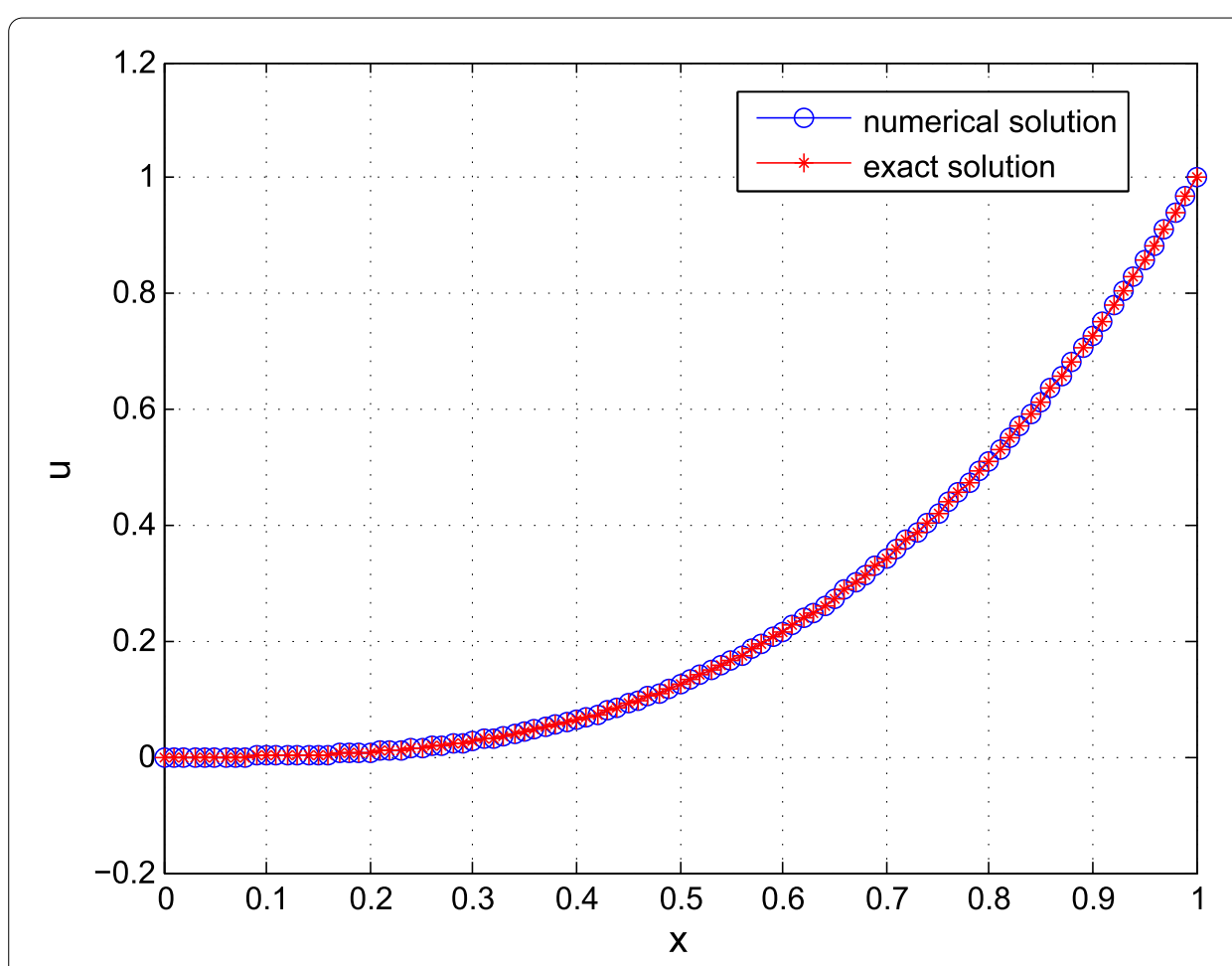

Figure 12 The numerical solution and the exact solution with $n=4$ for Example 2
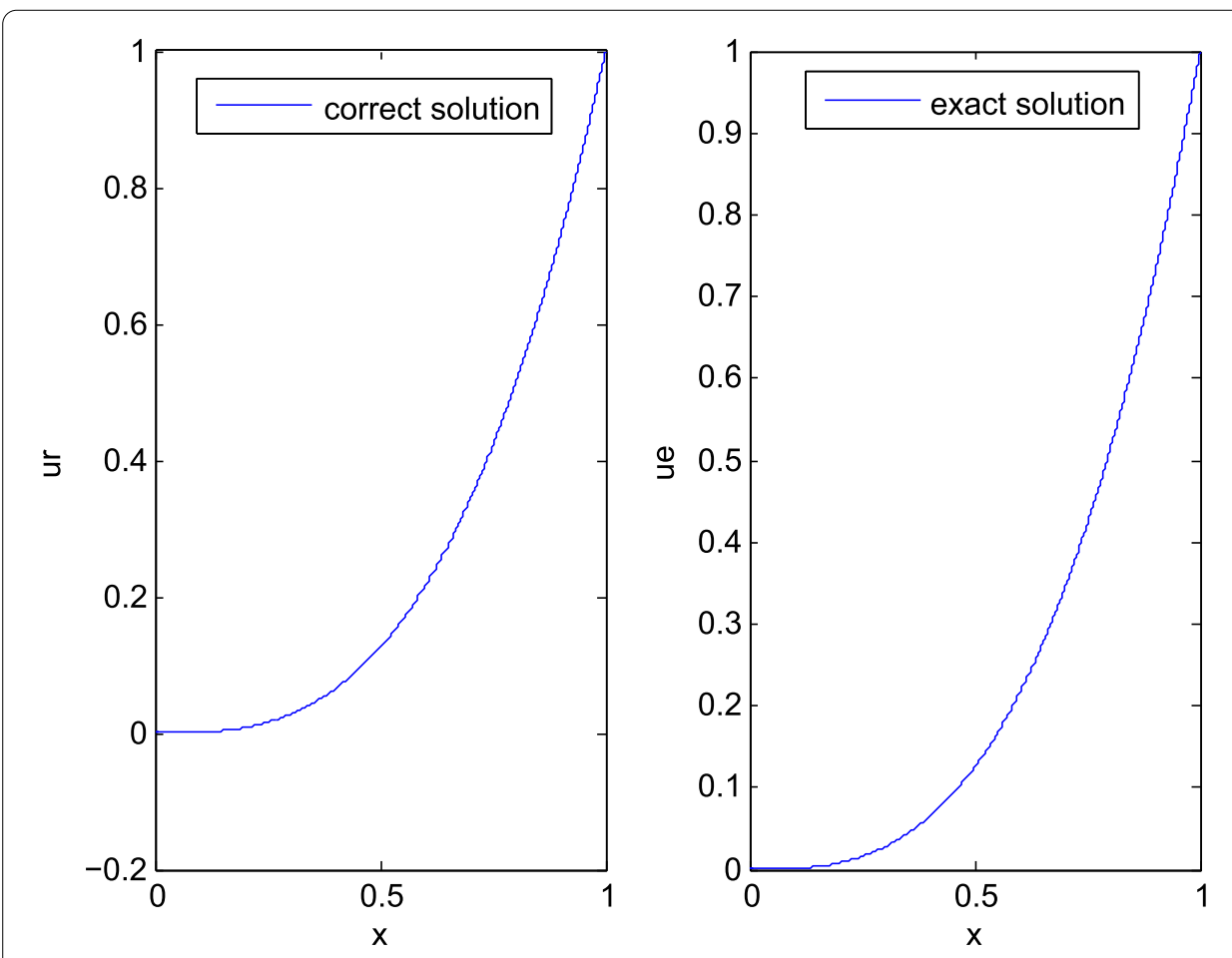

Figure 13 The correct solution and the exact solution with $n=2, m=4$ for Example 2 

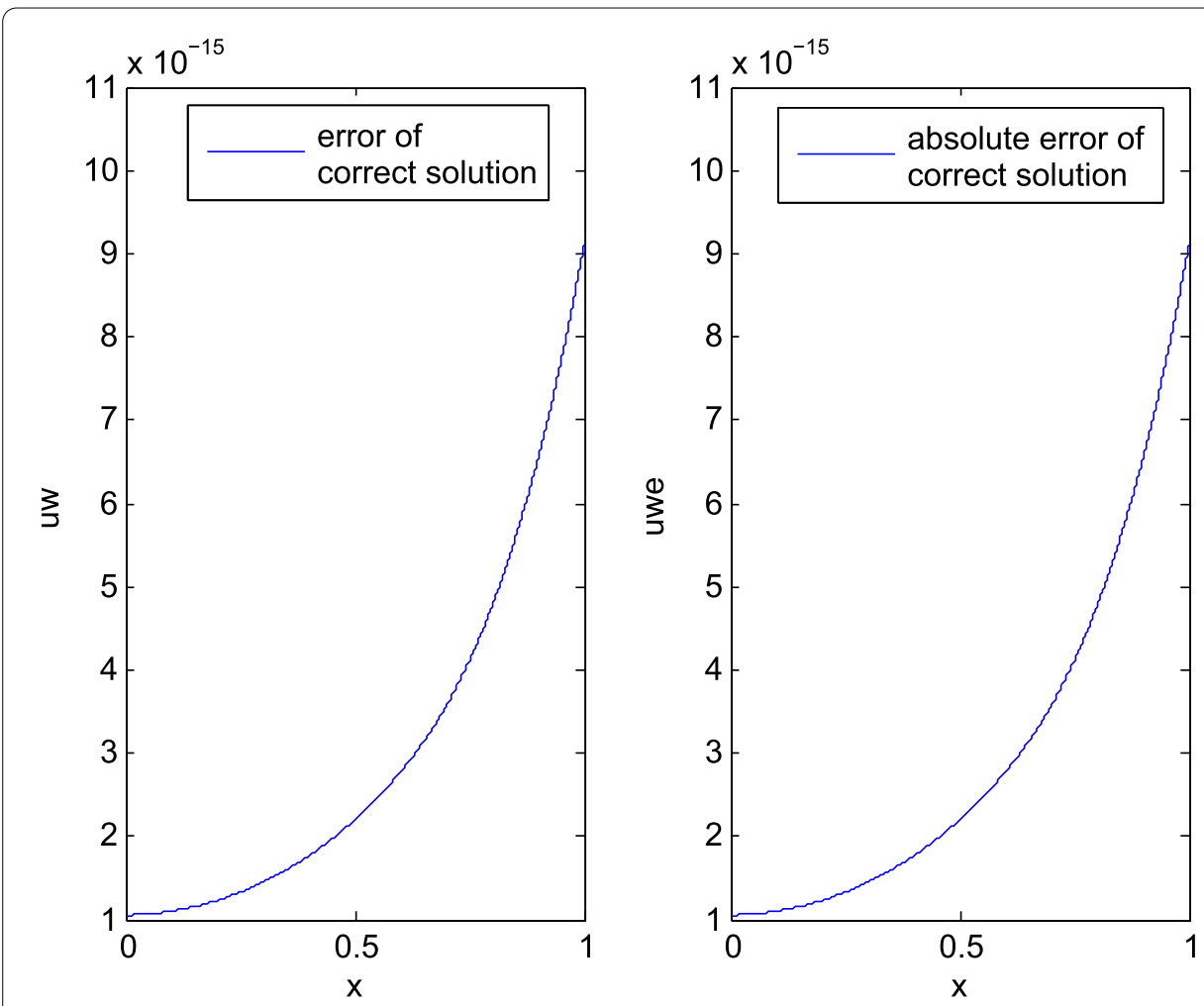

Figure 14 The error of correct solution and the absolute error of correct solution with $n=2, m=4$ for Example 2

tional differential operator matrix is deduced, but also this approach reduces the problem to a set of algebraic equations. We investigate multi-order fractional differential equation of error correction and analyze the convergence of the shifted Chebyshev polynomials. From example, it is seen that, $n$ is bigger, the absolute error is smaller, and the convergence effect between the numerical solution and the exact solution is better. We do the correction for the numerical solution, the absolute error of correct solution is smaller than the absolute error of numerical solution. Numerical experiments are given to demonstrate the applicability of the method and the validity of error correction.

\section{Acknowledgements}

The authors would like to thank the referees for careful reading and several constructive comments and for making some useful corrections that have improved the presentation of the paper. The authors would like to thank the School of

Science, Yanshan University, Qinhuangdao, Hebei, P.R. China for providing a good learning environment and INSA Centre Val de Loire, Université d'Orléans for offering to help.

Funding

This work is supported by the Natural Science Foundation of Hebei Province (A2017203100) in China and the LE STUDIUM RESEARCH PROFESSORSHIP award of Centre-Val de Loire region in France.

Availability of data and materials

Not applicable.

Competing interests

The authors declare that they have no competing interests.

Authors' contributions

The authors read and proved the final manuscript. 


\section{Author details}

'School of Sciences, Yanshan University, Qinhuangdao, P.R. China. ²Loire Valley Institute for Advanced Studies, Orléans, France. ${ }^{3}$ PRISME (INSA-Institut National des Sciences Appliquées)-88, Bourges, France. ${ }^{4}$ INSA Centre Val de Loire, Université d'Orléans, Bourges, France.

\section{Publisher's Note}

Springer Nature remains neutral with regard to jurisdictional claims in published maps and institutional affiliations.

Received: 28 January 2018 Accepted: 21 May 2018 Published online: 24 July 2018

\section{References}

1. Anastassiou, G.A., Argyros, I.K., Kumar, S.: Monotone convergence of extended iterative methods and fractional calculus with applications. Fundam. Inform. 151(1-4), 241-253 (2017)

2. Hajipour, M., Jajarmi, A., Baleanu, D.: An efficient nonstandard finite difference scheme for a class of fractional chaotic systems. J. Comput. Nonlinear Dyn. 13(2), 021013 (2017)

3. Jajarmi, A., Hajipour, M., Baleanu, D.: New aspects of the adaptive synchronization and hyperchaos suppression of a financial model. Chaos Solitons Fractals 99, 285-296 (2017)

4. Baleanu, D., Jajarmi, A., Hajipour, M.: A new formulation of the fractional optimal control problems involving Mittag-Leffler nonsingular kernel. J. Optim. Theory Appl. 175(3), 718-737 (2017)

5. Baleanu, D., Jajarmi, A., Asad, J.H., Blaszczyk, T.: The motion of a bead sliding on a wire in fractional sense. Acta Phys. Pol. 131(6), 1561-1564 (2017)

6. Kumar, D., Singh, J., Baleanu, D.: A new analysis of Fornberg-Whitham equation pertaining to a fractional derivative with Mittag-Leffler type kernel. Eur. Phys. J. Plus 133(2), 70 (2018). https://doi.org/10.1140/epjp/i2018-11934-y

7. Kumar, D., Singh, J., Baleanu, D.: Modified Kawahara equation within a fractional derivative with non-singular kernel. Therm. Sci. (2017). https://doi.org/10.2298/TSCI160826008K

8. Singh, J., Kumar, D., Hammouch, Z., Atangana, A.: A fractional epidemiological model for computer viruses pertaining to a new fractional derivative. Appl. Math. Comput. 316, 504-515 (2018)

9. Inc, M., Yusuf, A., Aliyu, A.I., Baleanu, D.: Soliton structures to some time-fractional nonlinear differential equations with conformable derivative. Opt. Quantum Electron. 50, 20 (2018)

10. Zeng, S., Baleanu, D., Bai, Y., Wu, G.: Fractional differential equations of Caputo-Katugampola type and numerical solutions. Appl. Math. Comput. 315, 549-554 (2017)

11. Ali, N., Shah, K., Baleanu, D., Arif, M., Khan, R.A.: Study of a class of arbitrary order differential equations by a coincidence degree method. Bound. Value Probl. 2017(1), 111 (2017)

12. Kumar, D., Singh, J., Baleanu, D.: A new numerical algorithm for fractional Fitzhugh-Nagumo equation arising in transmission of nerve impulses. Nonlinear Dyn. 91, 307-317 (2018)

13. Kumar, D., Agarwal, R.P., Singh, J.: A modified numerical scheme and convergence analysis for fractional model of Lienard's equation. J. Comput. Appl. Math. (2017) http://doi.org/10.1016/j.cam.2017.03.011

14. Sakar, M.G., Akgül, A., Baleanu, D.: On solutions of fractional Riccati differential equations. Adv. Differ. Equ. 2017(1), 39 (2017)

15. Jafarian, A., Mokhtarpour, M., Baleanu, D.: Artificial neural network approach for a class of fractional ordinary differential equation. Neural Comput. Appl. 28(4), 765-773 (2017)

16. Jafari, H., Lia, A., Tejadodi, H., Baleanu, D.: Analysis of Riccati differential equations within a new fractional derivative without singular kernel. Fundam. Inform. 151(1-4), 161-171 (2017)

17. Chen, Y.M., Han, X.N., Liu, L.C.: Numerical solution for a class of linear system of fractional differential equations by the Haar wavelet method and the convergence analysis. Comput. Model. Eng. Sci. 97(5), 391-405 (2014)

18. Chen, Y.M., Sun, L., Liu, L.L., Xie, J.Q.: The Chebyshev wavelet method for solving fractional integral and differential equations of Bratu-type. J. Comput. Inf. Syst. 9(14), 5601-5609 (2013)

19. Chen, Y.M., Sun, L., Li, X., Fu, X.H.: Numerical solution of nonlinear fractional integral differential equations by using the second kind Chebyshev wavelets. Comput. Model. Eng. Sci. 90(5), 359-378 (2013)

20. Chen, Y.M., Liu, L.Q., Li, B.F., Sun, Y.N.: Numerical solution for the variable order linear cable equation with Bernstein polynomials. Appl. Math. Comput. 238, 329-341 (2014)

21. Chen, Y.M., Sun, Y., Liu, L.Q.: Numerical solution of fractional partial differential equations with variable coefficients using generalized fractional-order Legendre functions. Appl. Math. Comput. 244, 847-858 (2014)

22. Baleanu, D., Inc, M., Yusuf, A., Aliyu, A.I.: Space-time fractional Rosenou-Haynam equation: Lie symmetry analysis, explicit solutions and conservation laws. Adv. Differ. Equ. 2018, 46 (2018)

23. Baleanu, D., Inc, M., Yusuf, A., Aliyu, A.l.: Time fractional third-order evolution equation: symmetry analysis, explicit solutions and conservation laws. J. Comput. Nonlinear Dyn. 13, 021011 (2018)

24. Firoozjaee, M.A., Yousefi, S.A., Jafari, H., et al.: On a numerical approach to solve multi order fractional differential equations with boundary initial conditions. J. Comput. Nonlinear Dyn. (2015)

25. Aphithana, A., Ntouyas, S.K., Tariboon, J.: Existence and uniqueness of symmetric solutions for fractional differential equations with multi-order fractional integral conditions. Bound. Value Probl. 2015(1), 1 (2015)

26. Hesameddini, E., Rahimi, A., Asadollahifard, E.: On the convergence of a new reliable algorithm for solving multi-order fractional differential equations. Commun. Nonlinear Sci. Numer. Simul. 34, 154-164 (2016)

27. Dabiri, A., Butcher, E.A.: Stable fractional Chebyshev differentiation matrix for the numerical solution of multi-order fractional differential equations. Nonlinear Dyn. 90(1), 185-201 (2017)

28. Saeedi, H.: A fractional-order operational method for numerical treatment of multi-order fractional partial differential equation with variable coefficients. SeMA J. 7, 1-13 (2017)

29. Dabiri, A., Butcher, E.A.: Numerical solution of multi-order fractional differential equations with multiple delays via spectral collocation methods. Appl. Math. Model. 56, 424-448 (2018)

30. Bolandtalat, A., Babolian, E., Jafari, H.: Numerical solutions of multi-order fractional differential equations by Boubaker polynomials. Open Phys. 14, 226-230 (2016) 
31. Pimenov, V.G., Hendy, A.S.: Numerical studies for fractional functional differential equations with delay based on BDF-type shifted Chebyshev approximations. Abstr. Appl. Anal. 2015(3), Article ID 510875 (2015)

32. Bhrawy, A.H., Alghamdi, M.A.: The operational matrix of Caputo fractional derivatives of modified generalized Laguerre polynomials and its applications. Adv. Differ. Equ. 2013(1), 307 (2013)

Submit your manuscript to a SpringerOpen ${ }^{\mathcal{O}}$ journal and benefit from:

- Convenient online submission

$\checkmark$ Rigorous peer review

- Open access: articles freely available online

- High visibility within the field

- Retaining the copyright to your article

Submit your next manuscript at $\gg$ springeropen.com 